\title{
Immunohistochemical Spectrum of GISTs at Different Sites and Their Differential Diagnosis with a Reference to CD117 (KIT)
}

\author{
Markku Miettinen, M.D., Leslie H. Sobin, M.D., Maarit Sarlomo-Rikala, M.D. \\ Department of Soft Tissue Pathology (MM) and Division of Gastrointestinal Pathology (LHS), Armed \\ Forces Institute of Pathology, Washington, District of Columbia, and Department of Pathology (MS-R), \\ Haartman Institute of the University of Helsinki, Helsinki, Finland
}

Gastrointestinal (GI) stromal tumor (GIST) is the designation for the major subset of GI mesenchymal tumors and encompasses most tumors previously classified as GI smooth muscle tumors. Although GISTs typically express CD117 (KIT), often express CD34, and sometimes express $\alpha$-smooth muscle actin (SMA), the relative frequency of these markers has not been characterized in large series of GISTs of different sites, and the CD1 17 expression has not been fully characterized in intra-abdominal tumors. In this study, we immunohistochemically analyzed 292 GISTs throughout the GI tract, including omentum and mesentery, and compared the immunoreactivities with 211 other tumors that may enter in the differential diagnosis. GISTs were defined in this study as CD117-positive primary spindled or epithelioid mesenchymal tumors of the GI tract, omentum, or mesentery. The CD34 positivity of GISTs varied from $47 \%$ in small bowel to 96 to $100 \%$ in rectum and esophagus, whereas SMA expression showed the opposite patterns and was most frequent in the GISTs of small bowel (47\%) and rarest in the GISTs of rectum and esophagus (10-13\%). Desmin was seen only occasionally. S100 positivity was rare but was seen most frequently in small intestinal GISTs (15\%). True leiomyomas from esophagus, muscularis mucosae of colorectum, and pericolic leiomyomas similar to uterine leiomyomas were negative for $\mathrm{CD117}$ and $\mathrm{CD34}$ and positive for SMA and desmin (46 of 46). Inflammatory fibroid polyps of stomach and small intestine

Copyright () 2000 by The United States and Canadian Academy of Pathology, Inc.

VOL. 13, NO. 10, P. 1134, 2000 Printed in the U.S.A.

Date of acceptance: May 9, 2000.

This work was supported by the American Registry of Pathology.

The opinions and assertions contained herein are the expressed views of

the authors and are not to be construed as official or as reflecting the views of the Departments of the Army or Defense.

Address reprint request to: Mankka Miettinen, M.D., Department of Soft

Tissue Pathology, Armed Forces Institute of Pathology, 14th Street and

Alaska Avenue, N.W., Washington, D.C. 20306-6000; fax:202-782-9182. were negative for $\mathrm{CD} 117$ but were often positive for CD34 (6 of 8) and variable for SMA (3 of 8). Inflammatory myofibroblastic tumors involving gastric or colonic wall were negative for $\mathrm{CD117}$ but some showed CD117-positive endothelia. GI schwannomas were all negative for $\mathrm{CD117}$ and positive for S100 protein (11 of 11). Extremely focal CD117 positivity was seen in the neoplastic cells of some retroperitoneal leiomyosarcomas and liposarcomas. Among other CD117-positive tumors were intestinal metastatic melanomas (8 of 11) and extraskeletal Ewing's sarcomas (5 of 11), two of which were abdominal. In conclusion, strong CD117 expression defines most primary GI mesenchymal tumors as GISTs, which show different patterns for CD34 and SMA in various parts of the GI tract. Some unrelated CD117-positive tumors (melanomas, Ewing's sarcomas) should not be confused with GISTs.

KEY WORDS: c-kit, Gastrointestinal stromal tumor, Genetics, Immunohistochemistry, Leiomyoma, Leiomyosarcoma, Pathology.

Mod Pathol 2000;13(10):1134-1142

Gastrointestinal (GI) stromal tumors (GISTs), previously classified as smooth muscle tumors, constitute the largest group of mesenchymal tumors of the stomach and small intestine. These tumors typically occur in older individuals and encompass the majority of tumors previously classified as cellular leiomyomas, leiomyoblastomas, and leiomyosarcomas of the GI tract, except in the esophagus, where typical leiomyomas are more common than GISTs (1-4). The GISTs are often positive for CD34, variably positive for smooth muscle actin, and usually positive for CD117 (KIT); the latter is probably the best defining feature for these tumors. Many GISTs, especially the malignant ones, also have activating mutations in the exon 11 of the c-kit gene that are believed to be pathogenetically important (5-15). 
The immunohistochemical features of GISTs of different sites have not been compared with adequate series, and the differential diagnostic implications of the possible heterogeneity of GISTs has not been systematically explored using a large series of tumors from different sites. Also, there are many GI and abdominal mesenchymal tumors that are unrelated to GISTs and may simulate them clinically, grossly, histologically, or by immunohistochemical patterns. To date, the number of such lesions studied for CD117 (KIT) remains relatively small, or comparative data are lacking altogether. Specific identification of GIST may become clinically important if therapies targeting the KIT tyrosine kinase activation become available.

The purpose of this article is to compare the immunohistochemical features of GISTs of different sites from esophagus to rectum to better delineate their immunohistochemical spectrum for the basis of diagnosis and differential diagnosis. We also evaluated the expression of CD117, CD34 smooth muscle actin, desmin, and $\mathrm{S} 100$ protein in a relatively large, representative selection of other intra-abdominal tumors or tumors that may present intra-abdominally and enter in the differential diagnosis of GIST.

\section{MATERIALS AND METHODS}

\section{Case Material}

Mesenchymal tumors involving the GI tract and coded as leiomyomas, leiomyosarcomas, smooth muscle tumors, or stromal tumors were retrieved from the files of the Haartman Institute of the University of Helsinki, Helsinki, Finland, from 1970 to 1998. A similar diagnostic selection from esophagus, colon, rectum, omentum, and mesentery was obtained from the files of the Soft Tissue and Gastrointestinal Pathology Registries of the Armed Forces Institute of Pathology. Additional tumor types studied for comparison included 40 retroperitoneal LMSs, 11 typical gastric schwannomas, and tumors, of which some were among those that CD117 positivity had been reported previously. These included 11 metastatic melanomas to intestines, 11 extraskeletal Ewing's sarcomas (two ab- dominal), and 5 intra-abdominal desmoplastic small round cell tumors (a total of 503 tumors).

\section{Diagnostic Definitions}

For this study, GISTs were defined as CD117 positive (more than $10 \%$ of tumor cells positive), spindle cell or epithelioid neoplasms primary in the GI tract, omentum, or mesentery, among the group of combined smooth muscle and stromal tumors. Excluded were other specific entities, such as histologically typical leiomyomas and leiomyosarcomas (see definition below), inflammatory myofibroblastic tumors, inflammatory fibroid polyps, desmoids, and schwannomas. Only GISTs of stomach and small intestine that had complete follow-up to allow for comparison between benign (no recurrence) and malignant tumors (intra-abdominal recurrence, liver metastases or both) were included. Spindle cell and epithelioid tumors were not separated, as previous studies showed them to be similar in their antigen expression (15).

GI leiomyomas were defined as benign tumors composed of well-differentiated smooth muscle cells, similar to those in the GI and vascular smooth muscle, and positive for $\alpha$-smooth muscle actin (SMA). GI leiomyosarcomas were defined as mitotically active tumors composed of spindled, welldifferentiated smooth muscle cells with bluntended nuclei and variably eosinophilic cytoplasm showing significant nuclear atypia, features similar to those of typical retroperitoneal and vascular leiomyosarcomas, and positivity for SMA.

\section{Immunohistochemistry}

The immunostains were performed using the avidin biotin peroxidase complex detection technique via an LSAB-kit (DAKO, Carpinteria, CA) or Vectastain Elite kit (Vector Labs, Burlingame, CA). Identical results were obtained in parallel experiments in a number of representative cases. Diaminobendizine containing hydrogen peroxide was used as the chromogen. The primary antibodies, dilutions, sources, and epitope retrieval modalities are listed in Table 1. Microwave epitope retrieval step was performed in an EDTA buffer $(372 \mathrm{mg} / \mathrm{L}$,

TABLE 1. Primary Antibodies Used in This Study and Their Dilution and Pretreatment Modalities and Commercial Source

\begin{tabular}{lcccc}
\hline \multicolumn{1}{c}{ Antigen } & Clone & Dilution & Pretreatment & Source \\
\hline CD117 (KIT) & Polyclonal & $1: 400$ & MW & Santa Cruz Biotechnology (Santa Cruz, CA) \\
CD34 & QBEND/10 & $1: 40$ & MW & Biogenex (San Ramon, CA) \\
$\alpha$-smooth muscle actin & 1 A4 & $1: 800$ & None & Sigma Chemicals (St. Louis, MO) \\
Desmin & D33 & $1: 40$ & None & DAKO (Carpinteria, CA) \\
S100 protein & Polyclonal & $1: 1600$ & None & DAKO \\
Estrogen receptor & 1D5 & $1: 40$ & DW & DAKO \\
\hline
\end{tabular}

MW, microwave. 
$\mathrm{pH}$ 8.0) for $20 \mathrm{~min}$, followed by a 20 -min cooling period before proceeding to blocking of endogenous peroxidase $(0.3 \%$ hydrogen peroxide in water), and to sequence of primary (60 $\mathrm{min})$, secondary (30 min), and tertiary reagent (30 min.), with peroxidase attachment (Avidin-Biotin block [Dako] was used in all cases).

The staining reactions were interpreted only in the presence of the following internal control in the target tissue to ensure the preservation of immunoreactivity in that tissue: CD117 (tissue mast cells or Cajal cells), CD34 (endothelial cells or perimuscular fibroblasts), SMA (vascular pericytes and smooth muscle), S100 protein (nerves or dendritic antigenpresenting cells). Such an internal control was not required for desmin, because positive cells (intestinal or large vessel smooth muscle) were not always present.

\section{RESULTS}

\section{GISTs}

The key demographic features and the patterns of immunoreactivity of 292 CD117-positive GISTs of different locations are summarized in Table 2. These tumors mainly occurred in older individuals, and the median age of patients with GISTs of all locations varied in a narrow range (59-64 yr). The male:female ratio significantly differed from 1:1 among the patients with esophageal (4.3), rectal (2.8), colonic (1.9), and small intestinal GISTs (1.41.5).

All GISTs were positive for CD117; this was used as part of the definition. The positivity typically appeared as diffuse cytoplasmic staining with common membrane accentuation but, in some cases, was focally perinuclear, 'golgi zone-like staining' (Fig. 1). Epithelioid GISTs tended to show less intense positivity as compared with the spindle cell GISTs. However, only seven cases showed CD117 positivity in less than $50 \%$ of tumor cells, and only one case had less than $20 \%$ CD117-positive cells.

The frequency of CD34 positivity varied significantly in GISTs of different locations. It was highest among the esophageal (100\%) and rectal rumors (92\%), whereas only $50 \%$ of the small intestinal GISTs were positive. The CD34 positivity was similar in benign and malignant gastric tumors (88$91 \%$ ), as defined by the follow-up data. There also was little difference in the CD34 expression between the benign and malignant small intestinal GISTs ( $47 \%$ versus $55 \%$ ).

The positivity for SMA varied according to location, and, in general, this marker showed an inverse pattern in frequency of expression, as compared with CD34. The highest frequency of SMA was seen among the small intestinal GISTs $(47 \%$ in the benign versus $27 \%$ in the malignant), whereas esoph-

TABLE 2. Demographic Features and Immunohistochemical Reactivities of KIT-positive GISTs by Locationa

\begin{tabular}{|c|c|c|c|c|c|}
\hline $\begin{array}{l}\text { Primary Site; Age Range (Median Age) } \\
\text { (yr); Male: Female Ratio }\end{array}$ & Total Cases & CD34 & SMA & Desmin & S100 Protein \\
\hline $\begin{array}{l}\text { Esophagus } \\
49-75(64) \\
\mathrm{M}: \mathrm{F}=4.3\end{array}$ & 16 & $\begin{array}{l}15 / 15 \\
(100)^{b}\end{array}$ & $\begin{array}{l}2 / 16 \\
(13)\end{array}$ & $\begin{array}{c}3 / 16 \\
(19)\end{array}$ & $\begin{array}{c}0 / 16 \\
(0)\end{array}$ \\
\hline $\begin{array}{l}\text { Stomach, serosal mininodules }(<1 \mathrm{~cm}) \\
60-88(65) \\
\mathrm{M}: \mathrm{F}=0.7\end{array}$ & 10 & $\begin{array}{l}9 / 10 \\
(90)\end{array}$ & $\begin{array}{l}0 / 10 \\
(0)\end{array}$ & $\begin{array}{l}0 / 10 \\
(0)\end{array}$ & $\begin{array}{c}0 / 10 \\
(0)\end{array}$ \\
\hline $\begin{array}{l}\text { Stomach, benign by follow-up } \\
21-87(64) \\
\mathrm{M}: \mathrm{F}=0.8\end{array}$ & 57 & $\begin{array}{c}52 / 57 \\
(91)\end{array}$ & $\begin{array}{c}16 / 57 \\
(28)\end{array}$ & $\begin{array}{c}3 / 57 \\
(5)\end{array}$ & $\begin{array}{c}0 / 57 \\
(0)\end{array}$ \\
\hline $\begin{array}{l}\text { Stomach, malignant by follow-up } \\
26-88 \text { (59) } \\
\mathrm{M}: \mathrm{F}=1.1\end{array}$ & 35 & $\begin{array}{c}29 / 33 \\
(88)\end{array}$ & $\begin{array}{c}12 / 35 \\
(34)\end{array}$ & $\begin{array}{c}0 / 33 \\
(0)\end{array}$ & $\begin{array}{c}2 / 33 \\
(6)\end{array}$ \\
\hline $\begin{array}{l}\text { Small bowel, benign by follow-up } \\
19-73(59) \\
M: F=1.4\end{array}$ & 30 & $\begin{array}{c}14 / 30 \\
(47)\end{array}$ & $\begin{array}{c}14 / 30 \\
(47)\end{array}$ & $\begin{array}{c}1 / 30 \\
(3)\end{array}$ & $\begin{array}{c}5 / 30 \\
(17)\end{array}$ \\
\hline $\begin{array}{l}\text { Small bowel, malignant by follow-up } \\
\text { 39-87 (59) } \\
\mathrm{M}: \mathrm{F}=1.5\end{array}$ & 30 & $\begin{array}{c}16 / 29 \\
(55)\end{array}$ & $\begin{array}{l}8 / 30 \\
(27)\end{array}$ & $\begin{array}{c}0 / 29 \\
(0)\end{array}$ & $\begin{array}{c}4 / 30 \\
(13)\end{array}$ \\
\hline $\begin{array}{l}\text { Colon } \\
28-82(63) \\
\text { M:F = } 1.9\end{array}$ & 20 & $\begin{array}{c}13 / 20 \\
(65)\end{array}$ & $\begin{array}{l}6 / 20 \\
(30)\end{array}$ & $\begin{array}{c}0 / 20 \\
(0)\end{array}$ & $\begin{array}{c}0 / 20 \\
(0)\end{array}$ \\
\hline $\begin{array}{l}\text { Rectum } \\
17-90(59) \\
\mathrm{M}: \mathrm{F}=2.8\end{array}$ & 71 & $\begin{array}{c}68 / 71 \\
(96)\end{array}$ & $\begin{array}{l}7 / 71 \\
(10)\end{array}$ & $\begin{array}{c}1 / 67 \\
(1)\end{array}$ & $\begin{array}{c}0 / 64 \\
(0)\end{array}$ \\
\hline $\begin{array}{l}\text { GIST of omentum and mesentery } \\
31-89(60) \\
\text { M:F }=1.1 \\
\text { Total of all sites }\end{array}$ & 292 tumors & $\begin{array}{c}14 / 22 \\
(64)\end{array}$ & $\begin{array}{c}13 / 23 \\
(57)\end{array}$ & $\begin{array}{c}3 / 22 \\
(14)\end{array}$ & $\begin{array}{c}0 / 23 \\
(0)\end{array}$ \\
\hline
\end{tabular}

GIST, gastrointestinal stromal tumor; SMA, $\alpha$-smooth muscle actin.

$a$ The benign and malignant tumors of the stomach and small intestine are grouped separately.

$b$ The percentage of positive cases is shown in brackets. 


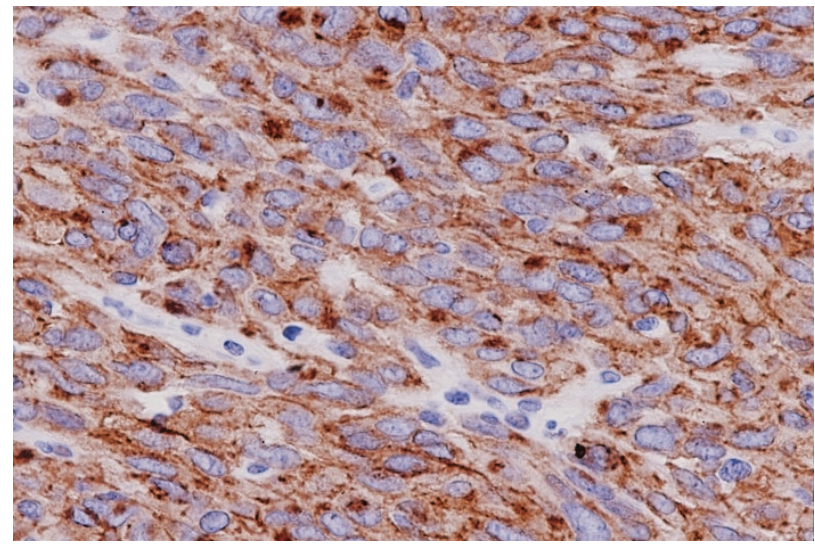

FIGURE 1. Gastrointerstinal stromal tumors typically show strong CD117 positivity and sometimes have perinuclear accentuation of staining.

ageal and rectal tumors were only rarely positive ( $13 \%$ and $14 \%$, respectively). Also, the small intestinal GISTs that were SMA positive, tended to be CD34 negative and vice versa. In many cases, the GISTs infiltrated within the fibers of the preexisting smooth muscle layer; such entrapped smooth muscle elements were not interpreted as part of the tumor.

Desmin positivity was rare and sporadic among the GISTs of all locations. It was seen in the esophageal and omental-mesenteric GISTs with the highest frequency (19\% and 14\%, respectively). Desmin positivity (usually focal) was only sporadically encountered in the benign gastric and small intestinal GISTs (5\% and 3\%) and was never seen in the malignant GISTs of stomach and small intestine.

S100 positivity was rare in the GIST group. However, in the present series, this marker was seen in $15 \%$ of the small intestinal tumors. The positivity was both cytoplasmic and nuclear and appeared in 10 to $100 \%$ of tumor cells.

\section{Leiomyomas}

Among these tumors, all GI leiomyomas were histologically distinctive from GISTs as paucicellular tumors with eosinophilic, often fibrillary, cytoplasm. These tumors, the intramural esophageal leiomyomas $(n=32)$ and the small polypoid leiomyomas of the muscularis mucosae of the colon and rectum $(n=22)$, were also immunohistochemically distinctive, as they were consistently negative for CD117, CD34, and S100 protein and positive for SMA and desmin. Numerous CD117positive mast cells were seen, especially in the esophageal leiomyomas, and to a lesser degree, in the leiomyomas of the muscularis mucosae of colon and rectum.

Another type of intra-abdominal leiomyoma that occurred on the external surface of the colon, and sometimes simulated GIST, was leiomyoma of the uterine type. All these 11 tumors occurred in women. They were variably cellular and showed alternating areas of collagen and cellular and focal myxoid zones resembling the uterine myomas. Immunohistochemically, they were consistently positive for SMA and desmin and negative for CD117 and CD34; mast cells were CD117 positive (Fig. 2). Most tumors (8 of 10) also were positive for estrogen receptor (ER) (Fig. 3). In comparison, none of the 20 randomly selected GISTs studied for ER for comparison, were positive.

\section{Leiomyosarcomas}

Among the gastric tumors, there were no examples of true leiomyosarcomas, whereas there were four small intestinal, four colonic, and two rectal tumors that histologically showed features of differentiated smooth muscle cells with blunt-ended nuclei and eosinophilic, sometimes granular, cytoplasm. These tumors were, by definition, all positive for SMA, and seven also were positive for desmin. Although all leiomyosarcomas were generally negative for CD117, scattered large neoplastic spindle cells (less than $1 \%$ of tumor cells) in two intestinal leiomyosarcomas showed strong cytoplasmic positivity; these four tumors were negative for CD34.

Among the 40 retroperitoneal leiomyosarcomas two showed scattered CD117-positive cells (Fig. 4). Twelve of 40 of the retroperitoneal tumors were positive for CD34; otherwise, these tumors showed similar features as the intestinal leiomyosarcoma by being consistently positive for SMA as a part of definition and usually positive for desmin (32 of 40; $80 \%)$. None of the CD34-positive retroperitoneal leiomyosarcomas were associated with stomach or intestines, and none showed GIST-like histologic features.

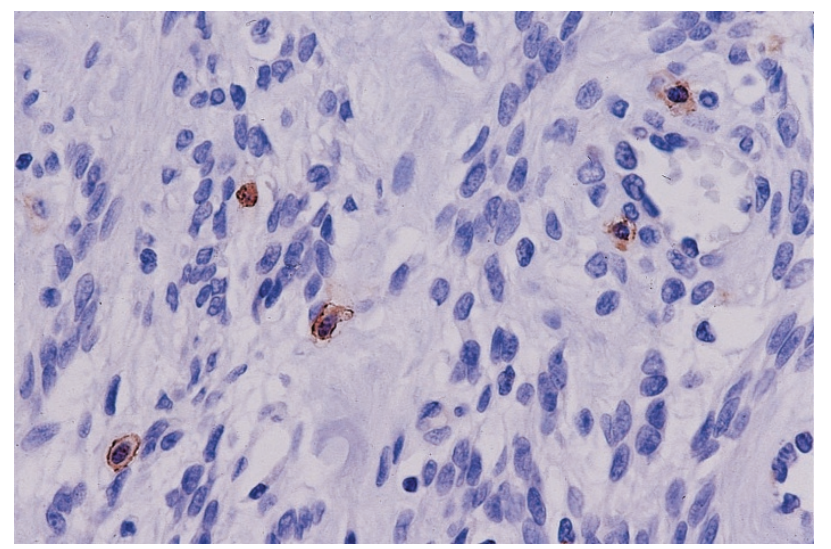

FIGURE 2. Uterine type pericolonic leiomyoma is negative for CD117, but mast cells are positive. 


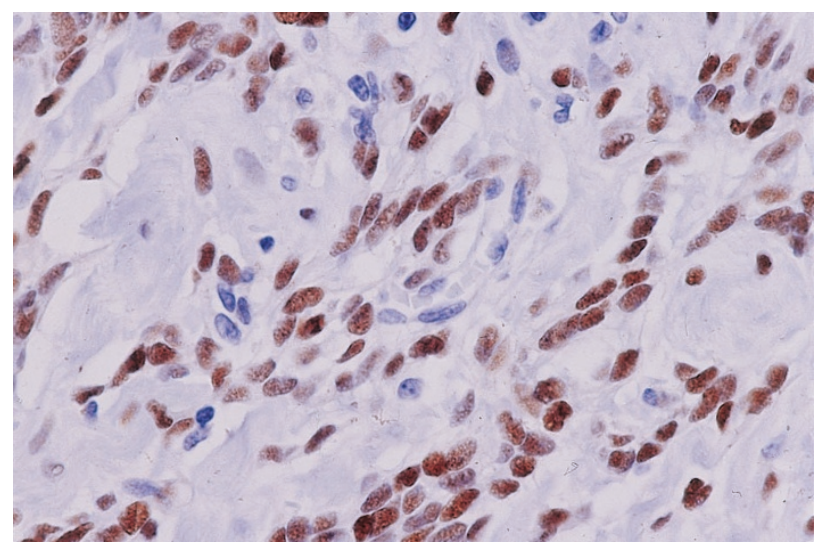

FIGURE 3. Uterine type leiomyoma also shows strong nuclear positivity for estrogen receptor.

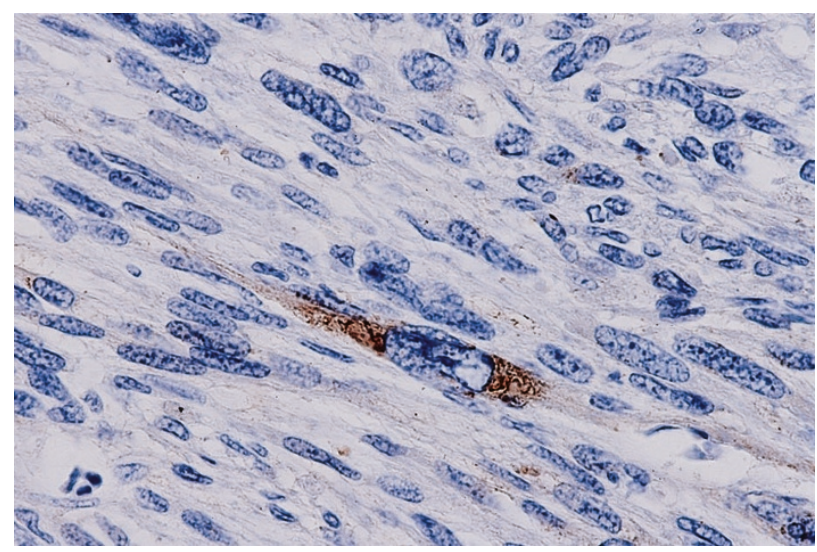

FIGURE 4. A retroperitoneal leiomyosarcoma show strong CD117positivity in sporadic tumor cells.

\section{Unclassified Sarcomas}

There were 17 primary GI tumors that were negative for CD117 and showed histologic similarities to GISTs. However, all were high-grade malignant spindle cell tumors, some of them with focal pleo- morphic features. Four of these tumors were from the stomach, one from the small bowel, six from the colon, and six from the rectum. The CD117negative tumors were all negative for CD34, desmin, and S100 protein. Three tumors showed focal SMA positivity; others were negative. These tumors were considered unclassified sarcomas based on the lack of definable differentiation.

\section{Patterns of Immunoreactivity for CD117 in Other Tumors}

The immunohistochemical studies of tumors that can be considered in the differential diagnosis of GIST either morphologically or based on CD117 positivity have been presented in Table 3 .

Inflammatory myofibroblastic tumors included all three childhood tumors in this study; four additional cases were seen in adults. Two of these tumors involved gastric, one duodenal, and four the colonic wall, often clinically or grossly simulating a GIST. All of these tumors were negative for CD117 and CD34; the latter marker showed high vascularity in these lesions. Two of seven cases showed unusual vascular endothelial positivity for CD117 (Fig. 5), whereas the lesional cells were negative for desmin and S100 but variably positive for SMA in four of seven cases.

Among CD34-positive tumors that were negative for CD117 were inflammatory fibroid polyps and peritoneal solitary fibrous tumors (Table 3 ). Inflammatory fibroid polyps (Fig. 6) were often positive and solitary fibrous tumors were consistently positive for CD34, whereas only mast cells were CD117 positive (Fig. 7). Liposarcomas occasionally showed CD117 positivity in sporadic tumor cells, similar to what was seen in four leiomyosarcomas.

The 11 schwannomas were spindle cell tumors, of which two occurred in the esophagus, seven in

TABLE 3. Immunohistochemical Patterns of Intestinal and Abdominal Tumor Other Than GISTs

\begin{tabular}{|c|c|c|c|c|c|}
\hline & CD117 & CD34 & SMA & Desmin & S100 Protein \\
\hline Leiomyoma of esophagus & $0 / 32$ & $0 / 32$ & $32 / 32$ & $32 / 32$ & $0 / 32$ \\
\hline Pericolonic leiomyoma of uterine type & $0 / 8$ & $0 / 8$ & $11 / 11$ & $11 / 11$ & $11 / 11$ \\
\hline Leiomyoma of muscularis mucosae & $0 / 22$ & $0 / 22$ & $22 / 22$ & $22 / 22$ & $0 / 22$ \\
\hline Leiomyosarcoma intestinal & $2 / 10^{a}$ & $0 / 10$ & $10 / 10$ & $7 / 10$ & $0 / 10$ \\
\hline Leiomyosarcoma retroperitoneal & $2 / 40^{a}$ & $12 / 40$ & $40 / 40$ & $32 / 40$ & $0 / 10$ \\
\hline Inflammatory myofibroblastic tumor & $0 / 7^{b}$ & $0 / 7$ & $4 / 7$ & $0 / 7$ & $0 / 7$ \\
\hline Inflammatory fibroidpolyp & $0 / 8$ & $6 / 8$ & $3 / 8$ & $0 / 8$ & $0 / 8$ \\
\hline Solitary fibrous tumor of abdomen & $0 / 5$ & $5 / 5$ & $0 / 5$ & $0 / 5$ & $0 / 5$ \\
\hline Desmoid of mesentery & $0 / 7$ & $0 / 7$ & $7 / 7$ & $4 / 7^{*}$ & $0 / 7$ \\
\hline Liposarcoma sclerosing & $2 / 7^{a}$ & $0 / 7$ & $0 / 7$ & $0 / 7$ & $0 / 7$ \\
\hline Liposarcoma, differentiated & $0 / 10$ & $0 / 10$ & $3 / 10$ & $1 / 10$ & $0 / 10$ \\
\hline Undifferentiated GI sarcoma & $0 / 17$ & $0 / 17$ & $3 / 17^{*}$ & $0 / 17$ & $0 / 17$ \\
\hline GI schwannoma & $0 / 11$ & $0 / 11$ & $0 / 11$ & $0 / 11$ & $11 / 11$ \\
\hline Metastatic melanoma to intestines & $8 / 11$ & $0 / 11$ & $0 / 11$ & $0 / 11$ & $11 / 11$ \\
\hline Extraskeletal Ewing's sarcoma & $5 / 11$ & $0 / 11$ & $0 / 11$ & $0 / 11$ & $0 / 11$ \\
\hline Desmoplastic small round cell tumor & $0 / 5$ & $0 / 5$ & $0 / 5$ & $5 / 5$ & $0 / 5$ \\
\hline Total & 211 & & & & \\
\hline
\end{tabular}

GIST, gastrointestinal stromal tumor; SMA, $\alpha$-smooth muscle actin.

$a$ Limited to focal staining in rare tumor cells.

$b$ Tumoral vascular endothelia were sometimes positive. 


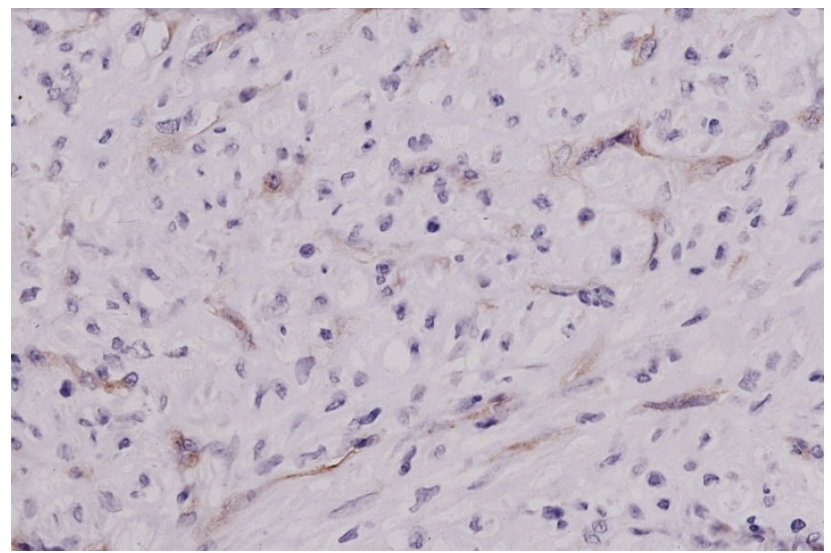

FIGURE 5. Inflammatory myofibroblastic tumor shows endothelial positivity for $\mathrm{CD} 117$, but the lesional myofibroblasts are negative.

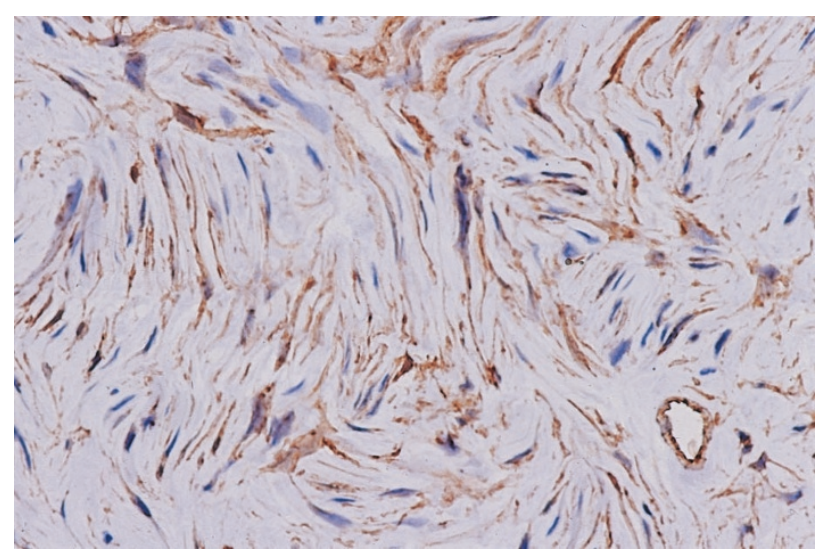

FIGURE 6. Inflammatory fibroid polyp shows CD34-positivity in the spindle cells in lamellar arrangement.

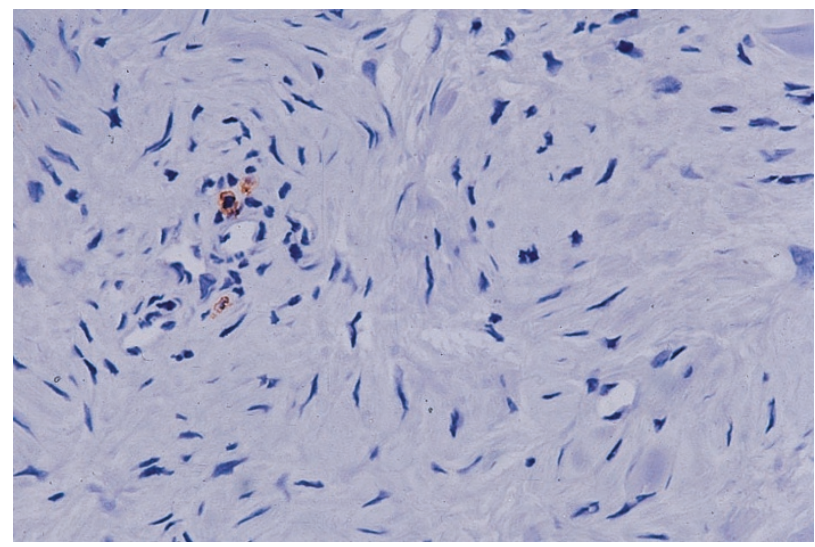

FIGURE 7. The tumor cells of inflammatory fibroid polyp are negative for CD117 but mast cells are positive.

stomach, and two in the colon. The clinicopathologic features of four gastric schwannomas were reported previously (16). These tumors often showed prominent lymphoid clusters in the periphery, sometimes with germinal centers. All tumors were negative for CD117, CD34, and muscle markers and were strongly positive for S100 protein.
Among small round cell tumors, CD117 positivity occurred in 5 of 11 extraskeletal Ewing's sarcomas, one of them intra-abdominal and another located in the abdominal wall (Fig 8). All five intraabdominal small round cell desmoplastic tumors were negative.

\section{DISCUSSION}

GIST is a designation that has been used recently for a specific group of gastrointestinal mesenchymal tumors, although this term occasionally has been used generically to refer to any mesenchymal tumors of the GI tract. In most instances, GISTs are understood as mesenchymal tumors essentially restricted to the GI tract. By their positivity for CD117 (KIT), they show phenotypic resemblance to the interstitial cells of Cajal of the GI tract. A unique feature for these tumors is the presence of activating mutations in the exon 11 of some c-kit genes, especially in the malignant tumors. Such mutations in the c-kit gene are a possible pathogenesis or explanation for the autonomous, unrestricted growth of malignant GISTs (2-11).

In this study, we analyzed more than 500 GI mesenchymal tumors to examine the immunohistochemical markers in the diagnosis and differential diagnosis of GISTs. For the purpose of this study, GISTs were exclusively defined as CD117positive mesenchymal spindle cell or epithelioid tumors primary in the stomach, intestines, or omentum and mesentery, among the tumors of the combined smooth muscle and stromal tumor group. By such definition, the vast majority of all mesenchymal tumors of the GI tract originally diagnosed as gastric or intestinal smooth muscle tumors or stromal tumors were defined as GISTs, except defined groups of benign leiomyomas of the esophagus and colon and rectum, and very small groups of differentiated leiomyosarcomas and undifferentiated sarcomas.

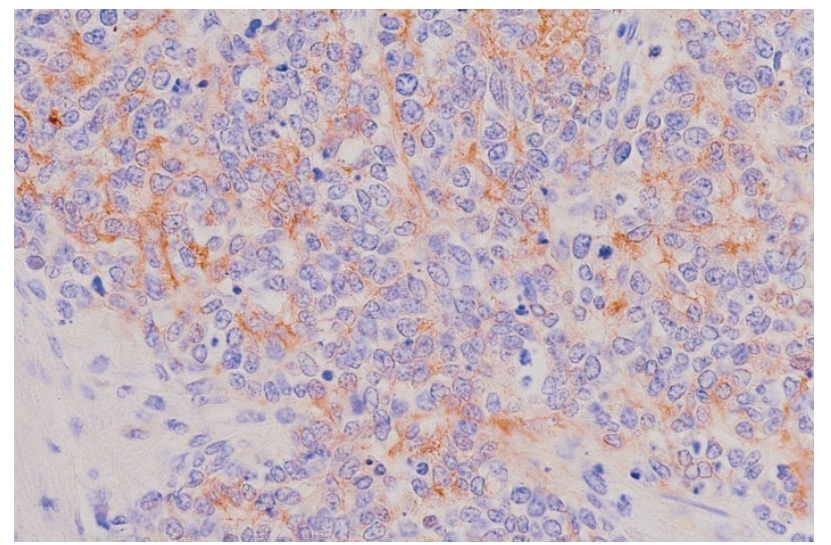

FIGURE 8. Intra-abdominal extraskeletal Ewing's sarcoma shows significant cytoplasmic CD117 positivity in the tumor cells. 
There are significant immunohistochemical differences in the GIST group in the frequency of CD34 and SMA expression, depending on the site of occurrence; this heterogeneity has not been appreciated fully in previous studies on GISTs (12-15, 17, 18). CD34 and SMA showed an opposite trend in expression in GISTs of several sites. Although most esophageal and rectal GISTs were positive for CD34, these tumors only rarely showed SMA. In contrast, small intestinal GISTs showed SMA as often as they showed CD34. The cause for this phenotypic heterogeneity and its significance are unclear. It is plausible that GISTs represent stem cell tumors that can differentiate into a Cajal cell-like or smooth muscle cell-like phenotype. Such a pluripotential GI mesenchymal stem cell population can differentiate both into smooth muscle and Cajal cells in the normal ontogeny, according to chimeric experiments on mice and birds of the chicken family $(19,20)$.

This study confirmed the previous impression that desmin expression is very rare in GISTs. Therefore, the simultaneous lack of desmin and presence of CD117 is a sharp contrast between GISTs and true smooth muscle tumors.

A small group of undifferentiated sarcomas was excluded from the defined GIST group. These tumors lacked CD117 and all other differentiation markers, including CD34, desmin, and S100 protein; focal actin positivity was occasionally encountered, and it cannot be ruled out that some of them are related to GISTs or that they represent undifferentiated leiomyosarcomas. We have encountered occasional undifferentiated tumors that lacked CD117 expression by immunohistochemistry but had c-kit mutations (10). Further correlative histologic, immunohistochemical, and molecular genetic studies are necessary to determine to what extent the CD117-negative cases with a null-cell phenotype belong to the biologic category of GISTs.

We also compared the immunohistochemical patterns of GIST with a large number of cases that included tumors originally considered to be smooth muscle tumors. This group includes the most important tumor entities that enter in the differential diagnosis of GIST. It especially includes tree leiomyomas and leiomyosarcomas and some inflammatory myofibroblastic tumors, inflammatory fibroid polyps, and nerve sheath tumors. Also, some CD117-positive unrelated tumors may occasionally enter in the differential diagnosis of GIST.

Leiomyomas occur in the GI tract in two forms: esophageal mural leiomyomas, and leiomyomas involving the muscularis mucosae layer of the colon and rectum. Common to all leiomyomas is their composition of histologically well-differentiated smooth muscle cells with eosinophilic cytoplasm. Indeed, immunophenotypically, these tumors fully correspond with smooth muscle tumors by showing smooth muscle actin and desmin and by being negative for CD117 and CD34. In this study, we did not encounter similar mural tumors in the intestines, suggesting that true mural leiomyomas of these locations must be rare, if they exist.

Another category of leiomyomas regularly found attached to the colon is the uterine type leiomyoma. These tumors do not only morphologically resemble uterine leiomyomas, but they also are positive for ER, which is similar to uterine leiomyomas $(21,22)$ and dissimilar to GISTs, which are ER-negative according to our results. Therefore, ER immunostaining is a valuable aid to separate the uterine type leiomyomas from GISTs.

True leiomyosarcomas that are composed of well-differentiated smooth muscle cells, similar to those seen in retroperitoneal and vascular LMSs, are vary rare in the GI tract. However, such tumors were encountered as a small minority among the malignant tumors, especially in the small intestine and colon. Although molecular genetic studies to date have shown lack of c-kit mutations in true LMSs, the number of cases studied remains small (10). Larger numbers of cases have to be analyzed comprehensively by combined histologic, immunohistochemical, and molecular studies to rule out the possibility that some tumors that express smooth muscle markers could be biologically related to the GIST group. Although LMSs were typically negative for CD117, a few cases showed extremely focal cytoplasmic staining in very occasional neoplastic cells. However, this pattern of staining is not likely to be confused with that of GISTs, because the latter typically show strong, almost always global, CD117 positivity.

Another unexpected feature found in this study was the moderately common CD34 expression in retroperitoneal LMSs (30\%). The significance of this expression is unclear at the present time but again illustrates the lower specificity of CD34 compared with CD117 for GISTs. An earlier study also reported CD34 expression in uterine and retroperitoneal leiomyosarcomas, in the latter with a frequency of $43 \%$ (23).

Schwannomas are rare mesenchymal tumors of the GI tract. They usually occur in the stomach but also may present in esophagus or colon $(16,24,25)$. These tumors are histologically distinctive as sharply demarcated spindle cell tumors, typically they are partially surrounded by a lymphoid cuff. They are strongly positive for S100 protein and always negative for CD117 and CD34. According to this study, there were no tumors that showed overlapping features between GIST and schwannoma. The S100 positivity found in a small group of GISTs, especially those of small intestine, is intriguing. It is best explained by multidirectional differentiation of 
these tumors; the lack of CD117 in schwannomas seems to allow for a sharp separation of GISTs and GI schwannomas.

Inflammatory myofibroblastic tumor, whose less cellular examples also have been called inflammatory pseudotumors and the more highly cellular and atypical examples have been called inflammatory fibrosarcoma $(26,27)$, occurs predominantly in childhood. One of the most common locations of this tumor is the abdomen, where the lesions can occur on the peritoneal surfaces and also involve the stomach and intestines. These tumors may simulate GISTs when involving the walls of stomach or intestines. However, they typically show a more heterogeneous cellular composition and are consistently negative for CD117 and CD34. The endothelial cells of this lesion seem to show CD117 immunoreacitvity similar to fetal and some malignant neoplastic endothelia; therefore, the tumor neovascularization in this lesion may be capable of similar reversion to fetal (CD117 positive) endothelial phenotype, as commonly seen in angiosarcomas (28).

Inflammatory fibroid polyps usually occur in stomach or small intestine as polyps. These tumors may occasionally resemble GISTs in a small biopsy because of their positivity for CD34 $(29,30)$. However, based on the present study, they are consistently negative for CD117, offering an immunohistochemical distinction from GIST.

Although liposarcomas typically have a prominent extra-intestinal component and reveal fatty differentiation in some areas, unless completely dedifferentiated, these tumors may also involve the intestinal walls, and then they simulate a primary GIST. The differential diagnosis is aided by the fact that tumors are negative for CD117, although focal cytoplasmic staining was occasionally seen in isolated cells. Such focal positivity is not likely to be confused with the GISTs, because GISTs typically show global CD117 positivity.

The CD117 (KIT) positive tumors that can involve the GI tract and occasionally be confused with GISTs include metastatic malignant melanoma, malignant vascular tumors, and metastatic pulmonary small cell carcinomas. Malignant melanoma may involve the stomach and especially small intestine, and occasionally such metastases may include GIST as a differential diagnostic consideration, although melanomas usually have patient history of the primary tumor. Like normal melanocytes, melanomas are often CD117 positive (31), and this study showed a surprisingly high frequency of CD117 positivity (73\%) among intestinal metastases of melanomas. Although melanocytes and primary melanomas are typically CD117 positive, it has been specifically shown that metastatic melanomas commonly have lessened CD117 expression, suggesting that this marker is lost in mel- anoma progression (32). An extremely rare event of occurrence of primary clear cell sarcoma (of tendons and aponeuroses) has been described in the small intestine (33). These sarcomas are CD117 positive in approximately $50 \%$ of cases and therefore may represent a diagnostic pitfall (6).

Other tumors that may be CD117 positive include malignant vascular tumors, especially angiosarcoma and occasionally Kaposi sarcoma (28); these diagnoses may be encountered in a mucosal biopsy of the GI tract. The presence of periodic acidSchiff-positive-hyaline globules in Kaposi sarcoma; vasoformation, at least to some degree, in angiosarcoma; and CD31 positivity in both of these tumors is diagnostically helpful, because GISTs are consistently CD31 negative (15).

This study revealed that some small round cell tumors, such as extraskeletal Ewing's sarcoma, may also be positive for CD117, although desmoplastic small round cell tumors that more commonly present intra-abdominally were negative. Although the clinicopathologic features of these tumors are usually sufficiently distinctive, such tumors (especially when intra-abdominal or in the abdominal wall) may occasionally enter in the differential diagnosis of GIST; some malignant GISTs show oval/ round cell cytologic pattern. A previous study also showed KIT expression in Ewing's family of tumors (34). Among small, round cell tumors in older adults, the major population of patients with GIST, metastatic pulmonary small cell carcinomas are often CD117 positive (29).

In summary, we have immunohistochemically evaluated 292 GISTs and more than 200 other tumors that enter in the differential diagnosis. GISTs, defined here as smooth muscle or stromal tumors that have CD117 expression, display different immunohistochemical profiles at various sites. This includes higher frequency of CD34 among esophageal and rectal tumors, a relatively high coexpression of SMA and CD34 in the small intestinal tumors, and inverse patterns of CD34 and SMA.

Although CD117 is quite specific for GISTs among primary mesenchymal tumors of the GI tract, limited CD117 expression may occur in unrelated tumors. Awareness of the immunohistochemical spectrum of GIST at different sites and the immunoreactivity patterns in tumors that have to be considered for differential diagnosis helps to precisely identify the GISTs and avoid the diagnostic pitfalls.

\section{REFERENCES}

1. Appelman HD: Mesenchymal tumors of the gut: histological perspectives, new approaches, new results, and does it make any difference? Monogr Pathol 31:220-46, 1990

2. Ueyama T, Guo K-J, Hashimoto H, Daimaru Y, Enjoji M: A 
clinicopathologic and immunohistochemical study of gastrointestinal stromal tumors. Cancer 69:947-55, 1992

3. Miettinen M, Sarlomo-Rikala M, Lasota J: Gastrointestinal stromal tumors: recent understanding of their biology. Hum Pathol 30:1213-20, 1999

4. Miettinen M, Sarlomo-Rikala M, Sobin LH, Lasota J: Esophageal stromal tumors: a clinicopathologic, immunohistochemical and molecular genetic study of seventeen cases and comparison with esophageal leiomyomas and leiomyosarcoma. Am J Surg Pathol 25:211-22, 2000

5. Kindblom LG, Remotti HE, Aldenborg F, Meis-Kindblom JM: Gastrointestinal pacemaker cell tumor (GIPACT). Gastrointestinal stromal tumors show phenotypic characteristics of the interstitial cells of Cajal. Am J Pathol 152:1259-69, 1998

6. Sarlomo-Rikala M, Kovatich A, Barusevicius A, Miettinen M: CD117: a sensitive marker for gastrointestinal stromal tumors that is more specific than CD34. Mod Pathol 11:72834, 1998

7. Sircar K, Hewlett BR, Huizinga JD, Chorneyko K, Berezin I, Riddell RH: Interstitial cells of Cajal as precursors for gastrointestinal stromal tumors. Am J Surg Pathol 23:377-89, 1999

8. Hirota S, Isozaki K, Moriyama Y, Hashimoto K, Nishida T, Ishiguro S, et al.: Gain-of-function mutations of c-kit in human gastrointestinal stromal tumors. Science 279:577-80, 1998

9. Kitamura Y, Hirota S, Nishida T: Molecular pathology of c-kit proto-oncogen and development of gastrointestinal stromal tumors. Ann Chir Gynaecol 87:282-6, 1998

10. Lasota J, Jasinski M, Sarlomo-Rikala M, Miettinen M: Mutations in exon 11 of c-kit occur preferentially in malignant versus benign gastrointestinal stromal tumors and do not occur in leiomyomas and leiomyosarcomas. Am J Pathol 154:53-60, 1999

11. Taniguchi M, Nishida T, Hirota S, Isozaki K, Ito T, Nomura T, et al.: Effect of c-kit mutation on prognosis of gastrointestinal stromal tumors. Cancer Res 59:4297-300, 1999

12. van de Rijn M, Hendrickson MR, Rouse RV: CD34 expression by gastrointestinal tract stromal tumors. Hum Pathol 25: 766-71, 1994

13. Mikhael AI, Bacchi CE, Zarbo RJ, Ma CK, Gown AM: CD34expression in stromal tumors of the gastrointestinal tract. Appl Immunohistochem 2:89-93, 1994

14. Monihan JM, Carr NJ, Sobin LH: CD34 immunoexpression in stromal tumours of the gastrointestinal tract and in mesenteric fibromatoses. Histopathology 25:469-73, 1994

15. Miettinen M, Virolainen M, Sarlomo-Rikala M: Gastrointestinal stromal tumors: value of CD34 antigen in their identification and separation from true leiomyomas and schwannomas. Am J Surg Pathol 19:207-16, 1995

16. Sarlomo-Rikala M, Miettinen M: Gastric schwannoma. Clinicopathologic analysis of six cases. Histopathology 27:33540, 1995

17. Franquemont DW, Frierson HF: Muscle differentiation and clinicopathologic features of gastrointestinal stromal tumors. Am J Surg Pathol 16:947-54, 1992

18. Rudolph P, Gloeckner K, Parvaresch R, Harms D, Schmidt D: Immunophenotype, proliferation, DNA-ploidy, and biological bahavior of gastrointestinal stromal tumors: A multivariate clinicopathologic study. Hum Pathol 29:791-800, 1998
19. Lecoin L, Gabella G, Le Douarin N: Origin of the c-kit positive interstitial cells in the avian bowel. Development 122: 725-33, 1996

20. Young HM, Ciampoli D, Southwell BR, Newgreen DF: Origin of interstitial cells of Cajal in the mouse intestine. Dev Biol 180:97-107, 1996

21. Sadan O, van Iddekinge B, van Gelderen CJ, Savage N, Becker PJ, van der Walt LA, et al.: Oestrogen and progesterone receptor concentrations in leiomyoma and normal myometrium. Ann Clin Biochem 24:263-7, 1987

22. Kawagichi K, Fujii S, Konishi I, Iwai T, Nanbu Y, Nonogaki H, et al.: Immunohistochemical analysis of oestrogen receptors, progesterone receptors and Ki67 in leiomyoma and myometrium during the menstrual cycle and pregnancy. Virchows Arch A Pathol Anat Histopathol 419:309-15, 1991

23. Rizeq MN, van de Rijn M, Hendrickson MR, Rouse RV: A comparative immunohistochemical study of uterine smooth muscle neoplasms with emphasis on the epithelioid variant. Hum Pathol 25:671-7, 1994

24. Daimaru Y, Kido H, Hashimoto H, Enjoji M: Benign schwannoma of the gastrointestinal tract: a clinicopathologic and immunohistochemical study. Hum Pathol 19:257-64, 1988

25. Prevot S, Bienvenu L, Vaillant JC, de Saint Maur P: Benign schwannoma of the digestive tract: a clinicopathologic and immunohistochemical study of five cases, including a case of esophageal tumor. Am J Surg Pathol 23:431-6, 1999

26. Meis-Kindblom JM, Kjellström C, Kindblom LG: Inflammatory fibrosarcoma: update, reappraisal, and perspective on its place in the spectrum of inflammatory myofibroblastic tumors. Semin Diagn Pathol 15:133-43, 1998

27. Coffin CM, Dehner LP, Meis-Kindblom JM: Inflammatory myofibroblastic tumor, inflammatory fibrosarcoma, and related lesions: an historical review with differential diagnostic considerations. Semin Diagn Pathol 15:102-10, 1998

28. Miettinen M, Lasota J: KIT expression in angiosarcomas and in fetal endothelial cells. Lack of c-kit mutations in exons 11 and 17 in angiosarcoma. Mod Pathol 13:536-41, 2000.

29. van De Rijn M, Hendrickson MR, Rouse RV: An immunohistochemical study of inflammatory fibroid polyps of the gastrointestinal tract. Appl Immunohistochem 2:54-9, 1994

30. Hasegawa T, Yang P, Kagawa N, Hirose T, Sano T: CD34 expression by inflammatory fibroid polyps of the gastrointestinal tract. Mod Pathol 10:451-6, 1997

31. Tsuura Y, Hiraki H, Watanabe K, Igarashi S, Shimamura K, Fukuda T, et al.: Preferential localization of c-kit product in tissue mast cells, basal cells of skin, epithelial cells of breast, small cell lung carcinoma and seminoma/dysgerminoma in human: immunohistochemical study of formalin-fixed, paraffin-embedded tissues. Virchows Arch 424:135-41, 1994

32. Montone KT, van Belle P, Elenitsas R, Elder DE: Protooncogene c-kit expression in malignant melanoma: protein loss with tumor progression. Mod Pathol 10:939-44, 1997

33. Ekfors T, Kujari H, Isomaki M: Clear cell sarcoma of tendons and aponeuroses (malignant melanoma of soft parts) in the duodenum: the first visceral case. Histopathology 22:255-9, 1993

34. Ricotti E, Fagioli F, Garelli E, Linari C, Crescenzio N, Horenstein AL, et al.: c-kit is expressed in soft tissue sarcoma of neuroectodermic origin and its ligand prevents apoptosis of neoplastic cells. Blood 91:2397-405, 1998 